\title{
Association between sucrose intake and risk of overweight and obesity in a prospective sub-cohort of the European Prospective Investigation into Cancer in Norfolk (EPIC-Norfolk)
}

\author{
Gunter GC Kuhnle ${ }^{1,2, *}$, Natasha Tasevska ${ }^{3}$, Marleen AH Lentjes ${ }^{2}$, Julian L Griffin ${ }^{4,5}$, \\ Matthew A Sims ${ }^{6}$, Larissa Richardson ${ }^{2}$, Sue M Aspinall ${ }^{2}$, Angela A Mulligan², \\ Robert $\mathrm{N}$ Luben $^{2}$ and Kay-Tee Khaw ${ }^{2}$ \\ 'Department of Food \& Nutritional Sciences, University of Reading, Reading RG6 6AP, UK: ${ }^{2}$ Department of Public \\ Health and Primary Care, University of Cambridge, Cambridge, UK: ${ }^{3}$ School of Nutrition and Health Promotion, \\ Arizona State University, Phoenix, AZ, USA: ${ }^{4}$ Department of Biochemistry, University of Cambridge, Cambridge, UK: \\ ${ }^{5}$ MRC Human Nutrition Research Unit, Cambridge, Cambridge, UK: ${ }^{6}$ MRC Epidemiology Unit, University of \\ Cambridge, Cambridge, UK
}

Submitted 23 September 2014: Final revision received 22 December 2014: Accepted 21 January 2015: First published online 23 February 2015

\begin{abstract}
Objective: The objective of the present study was to investigate associations between sugar intake and overweight using dietary biomarkers in the Norfolk cohort of the European Prospective Investigation into Cancer and Nutrition (EPIC-Norfolk).

Design: Prospective cohort study.

Setting: EPIC-Norfolk in the UK, recruitment between 1993 and 1997.

Subjects: Men and women ( $n$ 1734) aged 39-77 years. Sucrose intake was assessed using $7 \mathrm{~d}$ diet diaries. Baseline spot urine samples were analysed for sucrose by GC-MS. Sucrose concentration adjusted by specific gravity was used as a biomarker for intake. Regression analyses were used to investigate associations between sucrose intake and risk of BMI $>25.0 \mathrm{~kg} / \mathrm{m}^{2}$ after three years of follow-up.

Results: After three years of follow-up, mean BMI was $26 \cdot 8 \mathrm{~kg} / \mathrm{m}^{2}$. Self-reported sucrose intake was significantly positively associated with the biomarker. Associations between the biomarker and BMI were positive $(\beta=0.25$; $95 \%$ CI $0 \cdot 08,0 \cdot 43)$, while they were inverse when using self-reported dietary data $(\beta=-1.40 ; 95 \% \mathrm{CI}-1 \cdot 81$, -0.99). The age- and sex-adjusted OR for BMI $>25.0 \mathrm{~kg} / \mathrm{m}^{2}$ in participants in the fifth $v$. first quintile was $1.54\left(95 \% \mathrm{CI} 1.12,2 \cdot 12 ; P_{\text {trend }}=0.003\right)$ when using biomarker and 0.56 (95\% CI 0.40, 0.77; $P_{\text {trend }}<0 \cdot 001$ ) with self-reported dietary data.

Conclusions: Our results suggest that sucrose measured by objective biomarker but not self-reported sucrose intake is positively associated with BMI. Future studies should consider the use of objective biomarkers of sucrose intake.
\end{abstract}

Obesity and overweight are associated with increased risk for a number of chronic diseases, such as cancer ${ }^{(1)}, \mathrm{CVD}^{(2)}$ and type 2 diabetes. However, although energy balance is clearly central, there remains uncertainty about the role of specific dietary factors. While public perception suggests that the intake of sugar is associated with an increased risk of obesity and thus overweight people consume more sugar ${ }^{(3)}$, data from observational studies are inconsistent and weight increase or markers of obesity are associated mainly with the intake of sugar-sweetened beverages but not, or only to a small extent, with the total intake of sugar or sucrose ${ }^{(4,5)}$. Indeed, the US Institute of Medicine reported an inverse association between sugar intake and BMI in adults ${ }^{(6)}$. The
European Food Safety Authority based its scientific opinion on these findings ${ }^{(7)}$, although it omitted acknowledging that the Institute of Medicine considers the finding to be explained mainly by under-reporting. Under-reporting of diet has been found to be more prevalent among women and obese people $\mathrm{e}^{(8-10)}$ and it is mainly simple sugars and between-meal snacks that are most commonly underreported $^{(11)}$. This makes it difficult to interpret the inverse associations between reported sugar intake and BMI and to provide reliable recommendations to the public.

Urinary sugars, in particular sucrose and fructose, have been investigated and developed as dietary biomarkers of total sugar intake ${ }^{(12-16)}$. If $24 \mathrm{~h}$ urine collections are available, 
sucrose and fructose measured in $24 \mathrm{~h}$ urine can be used as predictive biomarkers of total sugar intake ${ }^{(16)}$. Given the sugars biomarker is a short-term measure of intake, when measured in spot urine its value will be associated with a certain amount of random error dependent on the timing of the spot urine collection. This error will be expected to attenuate the association between true intake and the biomarker. Nevertheless, earlier work showed sucrose in partial collections to be significantly correlated with sucrose intake $^{(13)}$. Previously, we have applied this biomarker to spot urine samples and investigated the association between sugar intake and obesity in a cross-sectional case-control study design in a sub-sample of the Norfolk cohort of the European Prospective Investigation into Cancer and Nutrition (EPIC-Norfolk), which only included normal weight $\left(B M I \leq 25.0 \mathrm{~kg} / \mathrm{m}^{2}\right)$ and obese $\left(\mathrm{BMI} \geq 30.0 \mathrm{~kg} / \mathrm{m}^{2}\right)$ participants ${ }^{(3)}$. In that study we found a significant positive association between the biomarker and obesity $(\mathrm{OR}=2 \cdot 44 ; 95 \%$ CI $1.54,3.86$ for the bottom $v$. top quintile).

Here, we prospectively investigated the association between sucrose intake and risk of overweight and obesity in a sample of the EPIC-Norfolk cohort study using urinary sugar biomarkers and self-reported dietary data.

\section{Materials and methods}

\section{Study population}

Between 1993 and 1997, approximately 77630 healthy men and women were invited to participate in the EPIC-Norfolk study through thirty-five medical practices in Norfolk ${ }^{(17,18)}$; 25639 participants, aged between 39 and 79 years, agreed to participate and attended the first health examination. Diet was assessed by $7 \mathrm{~d}$ diet diary (7DD) and a 130-item semiquantitative FFQ. The first day of the diary was completed as a $24 \mathrm{~h}$ recall (24HDR) with a trained interviewer, whereas the remainder was completed during subsequent days by the participants at home. Diary data were processed using the inhouse dietary assessment software DINER (Data Into Nutrients for Epidemiological Research ${ }^{(19)}$; data were checked and calculated using DINERMO, the software used to process data entered by DINER ${ }^{(20)}$. FFQ data were analysed using the in-house program, FETA (FFQ EPIC Tool for Analysis), to calculate the nutrient content ${ }^{(21)}$. Health and lifestyle characteristics were assessed by a questionnaire. Physical activity, representing occupational and leisure activity, was assessed using a validated questionnaire ${ }^{(22)}$, using four categorical variables (inactive, moderately inactive, moderately active and active). Height and weight measurements were collected following a standardised protocol as part of a health check conducted by trained research nurses $^{(23)}$. Spot urine samples were collected at baseline during the study visit (day 2 of the diary) and stored at $-20^{\circ} \mathrm{C}$ without preservatives. The study received ethical approval by the Norwich District Health Authority Ethics Committee and all participants gave signed informed consent.
Participants were invited back for a second health examination after three years of follow-up from 1997 to 2000 and 15786 participants attended. A health and lifestyle questionnaire was completed before the health examination. At the health examination, the protocol of the first health examination was repeated and data on height, weight and waist circumference (WC) were collected by trained nurses. The anthropometric measures collected at the second health examination were used as outcome measures in the analysis.

\section{Sample selection and missing data}

Baseline spot urine samples ( $n$ 5993) were selected randomly from the storage facility. While this selection was random, the samples are not necessarily a representative selection of the cohort. Co-variables (sex, dietary data and specific gravity) were missing for 155 participants and end points (anthropometric data) were missing for a further 2467 who did not attend the second health examination. Urinary sucrose analyses failed for 195 participants and results were outside the calibration range for 1442 participants, leaving a total sample size of 1734 (see Fig. 1 for details). For sensitivity analyses, sucrose concentrations below and above the limits of quantification $(<5.0 \mu \mathrm{m}$ or $>500 \mu \mathrm{m})$ were imputed with $4.9 \mu \mathrm{m}$ and $500 \cdot 1 \mu \mathrm{M}$, respectively.

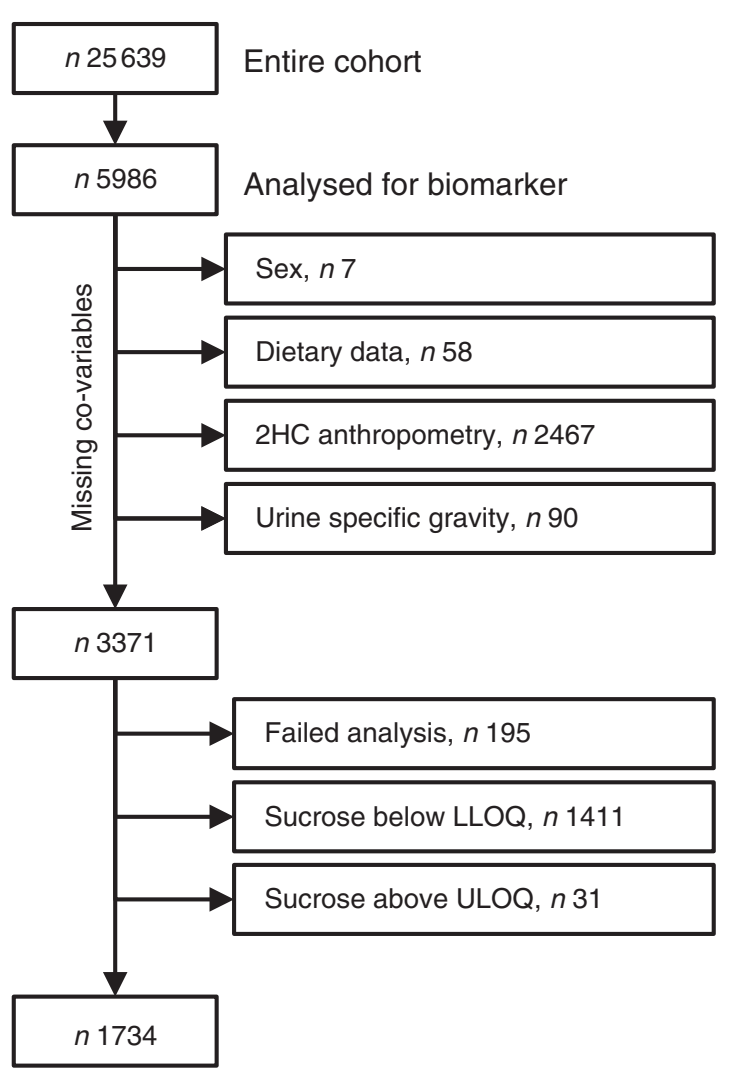

Fig. 1 Study population and sample size (2HC, second health check; LLOQ, lower limit of quantification; ULOQ, upper limit of quantification) 


\section{Analytical method}

Urinary sucrose and fructose were analysed using a modified version of the method described previously ${ }^{(24)}$. Urine aliquots $(200 \mu \mathrm{l})$ were mixed with $50 \mu \mathrm{l}$ internal standard solution $\left(\left[{ }^{13} \mathrm{C}_{6}\right]\right.$ fructose and $\left[{ }^{13} \mathrm{C}_{12}\right]$ sucrose, $100 \mu \mathrm{M}$ in water; CK Isotopes, Ibstock, UK) and $800 \mu \mathrm{l}$ cold acetonitrile was added to precipitate proteins. Samples were processed using a Hamilton Star (HAMILTON Robotics Ltd, Birmingham, UK) robot. The samples were mixed and centrifuged for $30 \mathrm{~min}$ at $14000 \mathrm{~g}$, then $500 \mu \mathrm{l}$ of the supernatant was transferred into a silanised glass vial and dried under reduced pressure. The samples were then derivatised as described previously ${ }^{(24,25)}$. Briefly, the samples were reconstituted in $30 \mu \mathrm{l}$ methoxyamine hydrochloride $(20 \mathrm{mg} / \mathrm{ml}$ in dry pyridine; Sigma-Aldrich, Gillingham, UK), mixed and incubated at room temperature for $30 \mathrm{~min}$. After $16 \mathrm{~h}$ at room temperature, $30 \mu \mathrm{l}$ $N$-methyl- $N$-trimethylsilyl-trifluoroacetamide containing $1 \%$ trimethylchlorosilane (Sigma-Aldrich) was added to each sample and incubated for $30 \mathrm{~min}$ at $75^{\circ} \mathrm{C}$. The derivatised samples were diluted with $540 \mu \mathrm{l}$ dry acetonitrile.

Samples were then analysed with a Trace GC Ultra and a Trace DSQ quadrupole mass spectrometer (ThermoElectron, Hemel Hempstead, UK). The derivatised sample was injected with a 1:10 split on to a $30 \mathrm{~m} \times 0.25 \mathrm{~mm}$ i.d., $5 \%$ phenylpolysilphenylene-siloxane column with a chemically bonded $25 \mathrm{~mm}$ TR-5MS stationary phase (ThermoElectron). The oven temperature was kept at $60^{\circ} \mathrm{C}$ for $2 \mathrm{~min}$ and then increased by $58^{\circ} \mathrm{C} / \mathrm{min}$ to $310^{\circ} \mathrm{C}$. The carrier gas was helium (flow rate $1.2 \mathrm{ml} / \mathrm{min}$ ). The mass spectrometer (transfer line temperature: $250^{\circ} \mathrm{C}$; ion source temperature: $275^{\circ} \mathrm{C}$; electron beam: $70 \mathrm{eV}$ ) was operated in full scan mode $(50-650 \mathrm{~m} / \mathrm{z} ; 3$ scans/s) and compounds were identified by their retention time and characteristic fragments.

Samples were quantified using the peak area ratio (analyte:internal standard) using an eight-point calibration line with samples prepared in water (concentration in $\mu$ м: 5, 10, 15, 25, 50, 75, 100 and 150). The difference between back-calculated and actual concentrations was always less than $5 \%$. Samples with a concentration outside the calibration range were diluted and re-analysed. Fructose was quantified using the sum of the peak area of the two epimers of the analyte and the internal standard. Quality control samples were prepared by adding known amounts of sucrose and fructose to spot urine samples and including at least three (with low, medium and high concentrations) in each analytical batch. Supplemental Table 1 (see online supplementary material) shows the reproducibility of the method for urinary sucrose and fructose at different concentrations. Urinary sucrose and fructose concentration remained stable for at least $7 \mathrm{~d}$ at $4^{\circ} \mathrm{C}$.

The STROBE (Strengthening the Reporting of Observational Studies in Epidemiology) checklist for cohort studies ${ }^{(26)}$ has been completed for the present study.

\section{Data analysis}

For data analyses, samples with urinary sucrose concentrations outside the acceptable quantification range were excluded. Based on the data shown in Supplemental Table 1 , the acceptable range for sucrose was $5 \mu \mathrm{m}$ to $500 \mu \mathrm{M}(1.7 \mathrm{mg} / \mathrm{l}$ to $171 \mathrm{mg} / \mathrm{l})$ and $20 \mu \mathrm{m}$ to $500 \mu \mathrm{m}(3.6 \mathrm{mg} / \mathrm{l}$ to $90 \mathrm{mg} / \mathrm{l})$ for fructose. Data on urinary fructose were used in the sensitivity analysis only, whereas the main analysis was conducted using urinary sucrose as a biomarker for sucrose intake. Concentration of sucrose in urine was expressed relative to specific gravity to adjust for urine concentration. Creatinine could not be used, given the highly significant association between urinary creatinine and BMI $(\rho=0 \cdot 15 ; P<0 \cdot 0001)$.

The distributions of the biomarker (urinary sucrose) and self-reported sugar intake were skewed and therefore all analyses with continuous data were conducted with logtransformed data. Both sex-specific and sex-adjusted analyses were conducted. 7DD sucrose intake was adjusted for energy intake using the nutrient density method $(\mathrm{g} / \mathrm{MJ})$. The association of self-reported energyadjusted sucrose intake or sucrose biomarker with BMI and WC at the second health examination was assessed using linear regression models adjusted for age and sex; for WC, the model was also adjusted for height at the second health check. We report $\beta$ coefficients for the regression of BMI or WC $v$. log-transformed biomarker or self-reported energy-adjusted sucrose intake. Participants were divided into quintiles by urinary biomarker (sucrose adjusted by specific gravity) and dietary intake (selfreported sucrose intake, 7DD). Odds ratios and 95\% confidence intervals for BMI $>25.0 \mathrm{~kg} / \mathrm{m}^{2}$ after three years of follow-up were estimated using unconditional logistic regression in age- and sex-adjusted models. Tests for linear trend were conducted by treating quintiles as continuous variables.

We also included biomarker (log-transformed) and selfreported intake (7DD, energy-adjusted, log-transformed) in the same linear regression model to calculate adjusted means of BMI (additionally adjusted for age) and WC (additionally adjusted for age and height) after three years of follow-up.

Statistical analyses were conducted with the statistical software package Stata version 11.2 and $\mathrm{R}^{(27)}$. The $P$ values for statistical tests were two-tailed and considered statistically significant at a level of less than $0 \cdot 05$.

\section{Results}

\section{Study population}

Spot urine samples were analysed from a selection of 5986 participants (2578 men, $43 \%$; 3408 women, $57 \%$ ) of EPICNorfolk. Co-variables and end points were available for 3371 participants (1338 men, 41\%; 1983 women, 59\%; Fig. 1). Urinary sucrose concentration was outside the 
calibration range $(5 \mu \mathrm{m}$ to $500 \mu \mathrm{m})$ or could not be detected due to analytical problems in 1637 participants, leaving 1734 participants (797 men, 46\%; 937 women, 54\%) for whom biomarker data were available. After three years of follow-up, the mean BMI increased from 26.2 (95\% CI $26 \cdot 0,26 \cdot 4) \mathrm{kg} / \mathrm{m}^{2}$ at baseline to (95\% CI 26.6, 27.0) $26.8 \mathrm{~kg} / \mathrm{m}^{2}$, and $35 \%, 48 \%$ and $17 \%$ of the participants were normal weight, overweight and obese, respectively. The mean WC was $96.9(95 \%$ CI $96 \cdot 2,97 \cdot 6) \mathrm{cm}$ in men and $82 \cdot 1$ (95\% CI $81 \cdot 5,82 \cdot 8) \mathrm{cm}$ in women.

We compared urinary sucrose, adjusted by specific gravity, as a biomarker for sucrose intake with energyadjusted, self-reported 7DD sucrose intake. Supplemental Table 2 gives a summary of the baseline characteristics of study participants, divided into quintiles by biomarker and self-reported intake, respectively (more details are shown in Supplemental Tables 3 and 4, see online supplementary material). Independent of the classification method used (biomarker or energy-adjusted self-reported intake), age, energy and sucrose intake (g/d) increased across quintiles. The proportion of dietary sucrose to total dietary sugars also increased from the bottom to the top quintile, with a larger range being observed when using self-reported dietary data. However, while there were more women in the bottom quintile of biomarker and more men in the top one, this relationship was reversed when using selfreported dietary data. Mean BMI measured at both baseline and the second health check increased across quintiles for the biomarker, while it decreased across quintiles for self-reported sucrose. Similar observations were made for WC measured at the second health check.

\section{Association between intake and biomarker}

In sex-combined analysis, energy-adjusted intake of sucrose (7DD) was significantly associated with the biomarker $(\beta=0.078 ; 95 \%$ CI $0.059,0.097)$ and this association did not change materially when adjusted for age and sex. In men, the association was considerably stronger $(\beta=0 \cdot 108 ; 95 \%$ CI $0 \cdot 079,0 \cdot 138)$ than in women $(\beta=0 \cdot 060$; $95 \%$ CI $0.035,0 \cdot 085)$. This association was also significant for non-energy adjusted sucrose intake $(\beta=0 \cdot 188 ; 95 \% \mathrm{CI}$ $0 \cdot 144,0 \cdot 232$; per $25 \mathrm{~g} / \mathrm{d}$ increase in sucrose intake) and remained stable after adjusting for age and sex. As above, the association was stronger in men $(\beta=0.231 ; 95 \% \mathrm{CI}$ $0 \cdot 155,0 \cdot 306$; per $25 \mathrm{~g} / \mathrm{d}$ increase in sucrose intake) than in women ( $\beta=0.094 ; 95 \%$ CI $0.045,0.143$; per $25 \mathrm{~g} / \mathrm{d}$ increase in sucrose intake).

The relationship between biomarker and self-reported intake, expressed as the ratio of biomarker to energyadjusted sucrose intake, was positively associated with baseline and follow-up BMI (Fig. 2). Indeed, there was a significant positive association with BMI at follow-up $(\beta=0.04 ; 95 \%$ CI $0.03,0.05)$ in an unadjusted model and after adjusting for age and sex $(\beta=0.04 ; 95 \%$ CI 0.02 , $0 \cdot 05)$. The median ratio was approximately $50 \%$ higher in overweight and obese participants when compared with normal-weight participants.

\section{Associations between self-reported sucrose intake, biomarker, BMI and waist circumference}

Table 1 shows the associations between sucrose, determined either by biomarker or 7DD, and BMI and WC at follow-up. The data show a positive association for the biomarker with BMI and WC, but an inverse association for both energy-adjusted ( $\beta=-1.40 ; 95 \% \mathrm{CI}-1 \cdot 81,-0 \cdot 99)$ and absolute $(\beta=-1.38 ; 95 \%$ CI $-1.72,-1.04)$ selfreported intake (adjusted for age and sex). These associations were statistically significant for women with both biomarker and 7DD data, but only with 7DD data for men. When including biomarker and self-reported dietary data in the same model, the associations were strengthened and remained in opposite directions (biomarker, logtransformed: $\beta=0.38 ; 95 \%$ CI $0.21,0.55$; dietary data, energy-adjusted and log-transformed: $\beta=-1.57$; $95 \% \mathrm{CI}$ $-1 \cdot 99,-1 \cdot 16$; Table 1). Similar relationships were found after stratification by sex (data not shown).

\section{Associations between self-reported sucrose intake, biomarker and risk of being overweight}

There were significant associations between the biomarker and risk of being overweight or obese after three years of follow-up (Table 2, Fig. 3) with an OR of 1.54 (95\% CI 1.12, 2.12; $P=0.008)$ between the top and bottom quintile and a significant trend $(P=0.003)$ across quintiles. Stratification by sex showed a marginally non-significant trend $(P=0.054)$ in men and a significant $(P=0.02)$ trend in women. Conversely, there was an inverse association when using selfreported intake with an OR of 0.56 (95\% CI 0.40, 0.77; $P<0.0001)$ and also a significant trend $(P<0 \cdot 0001)$. After stratification for sex, the trend remained significant in both men and women. When using the biomarker as a continuous variable, the OR for $\mathrm{BMI}>25.0 \mathrm{~kg} / \mathrm{m}^{2}$ was 1.16 (95\% CI $1.05,1.27)$ per log increase. Conversely, the OR was 0.60 (95\% CI $0.47,0.77$ ) when using self-reported dietary data. An analysis of the association between intake and risk of being overweight or obese at baseline gave similar results in the sex-adjusted model (see online supplementary material, Supplemental Table 5).

The ratio of urinary sucrose to fructose concentration has been used previously as a biomarker of sugar intake in relation to risk of being overweight ${ }^{(3)}$. Applying this biomarker in the current study reduced the sample size, given there were fewer available samples with both sucrose and fructose values within the acceptable analytical range, to 1238 participants ( 578 men, $47 \%$; 660 women, $53 \%$ ). The associations observed with urinary sucrose to fructose ratio were not materially different from those observed with specific-gravity adjusted sucrose concentration in urine (see online supplementary material, Supplemental Table 6). 


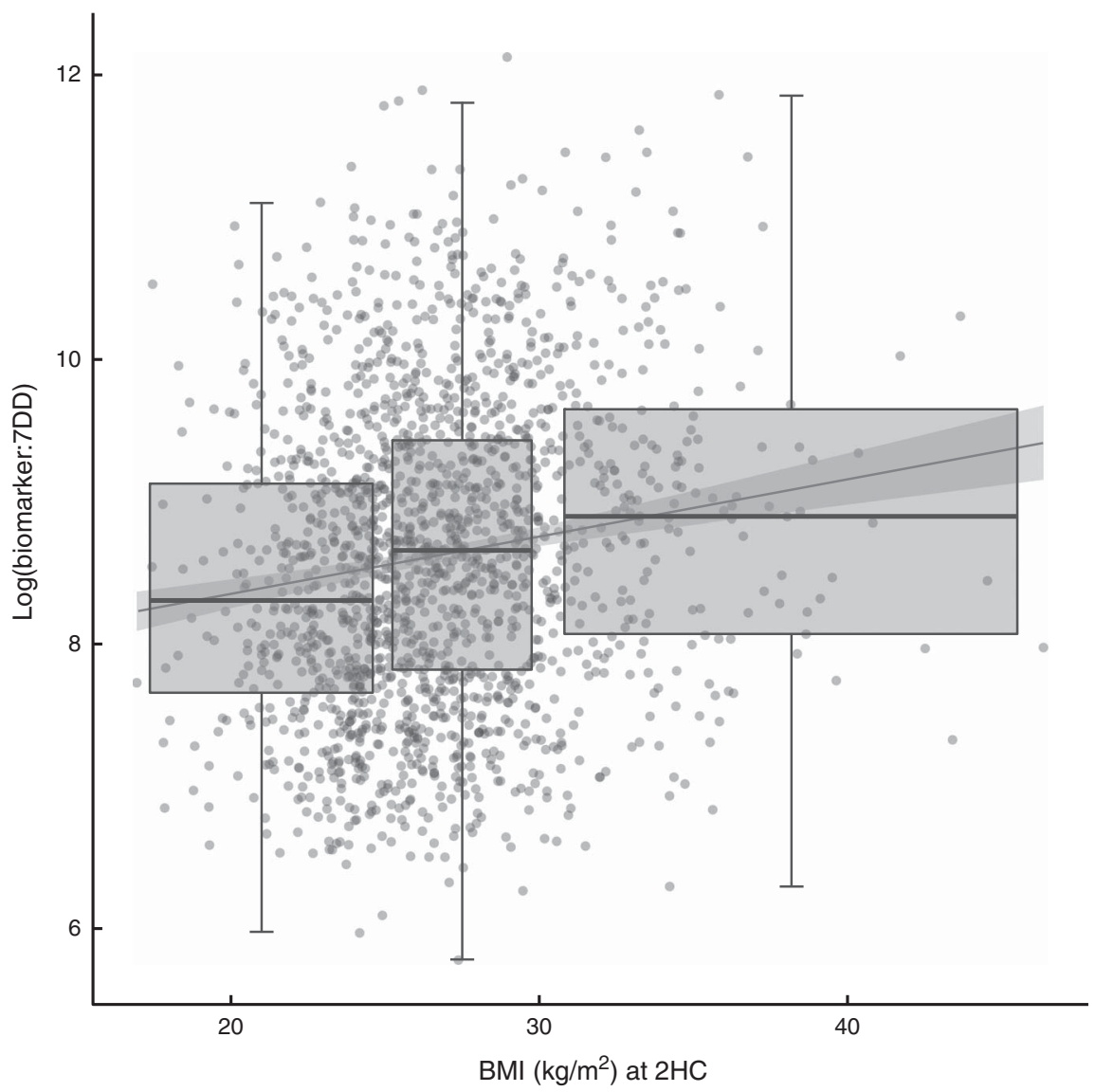

Fig. 2 Relationship between the biomarker (specific-gravity-adjusted urinary sucrose) and self-reported intake (energy-adjusted sucrose intake, as assessed by $7 \mathrm{~d}$ diet diary (7DD)), expressed as a ratio, and BMl after three years of follow-up among men and women ( $n$ 1734) aged 39-77 years, Norfolk cohort of the European Prospective Investigation into Cancer and Nutrition (EPICNorfolk). Presented are box-and whisker plots in which the bottom and top of the box represents the 25th and 75th percentile, respectively (the interquartile range), the line within the box represents the median and the bottom and top of the whisker represents the minimum and maximum value, respectively, of log-transformed ratio of biomarker to 7DD for three BMI classes (normal weight (left), overweight (middle) and obese (right)) at the second health check (2HC); and a least-square linear model with $95 \%$ confidence interval $(\square)$

\section{Comparison of different dietary assessment instruments ( $7 \mathrm{~d}$ diet diary, $24 \mathrm{~b}$ recall and $\mathrm{FFQ}$ )} Energy-adjusted sucrose intake by $24 \mathrm{HDR}$ (first day of 7DD) was significantly associated with estimates from 7DD $(\beta=1.08 ; 95 \%$ CI $1.04,1.13)$ and the biomarker ( $\beta=0 \cdot 11 ; 95 \%$ CI 0.08, 0.14; age- and sex-adjusted model). The latter association was stronger in men than in women (data not shown).

FFQ-measured sucrose (g/MJ), available for 1685 participants, was significantly associated with sucrose measured by both 7DD $(\beta=0.70 ; 95 \%$ CI $0.65,0.75)$ and 24HDR $(\beta=0.88$; $95 \%$ CI $0.81,0.96)$. The association with the biomarker was also significant $(\beta=0.05$; $95 \%$ CI 0.04, 0.07; age- and sexadjusted model) and stronger in men than in women.

Self-reported sucrose intake, independent of the dietary assessment instrument used and adjustment for energy intake, was inversely associated with BMI and WC after three years of follow-up (Table 3), although the associations were weakest with data from $24 \mathrm{HDR}$.

\section{Effect of physical activity}

The associations observed did not change materially after including physical activity in the model (see online supplementary material, Supplemental Table 7).

\section{Sensitivity analyses with biomarker data outside the calibration range}

Biomarker data were available for 3176 participants (1297, $41 \%$ men; 1879, 59\% women; see online supplementary material, Supplemental Table 8 for details), although the urinary sucrose concentration in 1411 participants was below and in thirty-one participants was above the limit of quantification. In men, baseline BMI of participants with urinary sucrose concentration below the limit of quantification ( $n$ 498; mean $\mathrm{BMI}=26.2 \mathrm{~kg} / \mathrm{m}^{2}$ ) was significantly $(P=0.04, t$ test) lower than that of participants with sucrose concentration within the calibration range $(n 821$; mean $\left.\mathrm{BMI}=26.5 \mathrm{~kg} / \mathrm{m}^{2}\right)$. No significant difference was observed in women $(P=0.65)$. 
Table 1 Associations between sucrose intake (by biomarker or $7 \mathrm{~d}$ diet diary (7DD)), BMI and waist circumference (WC) after three years of follow-up at the second health check among men and women ( $n$ 1734) aged 39-77 years, Norfolk cohort of the European Prospective Investigation into Cancer and Nutrition (EPIC-Norfolk)

\begin{tabular}{|c|c|c|c|c|c|c|c|c|c|c|c|c|}
\hline & \multicolumn{6}{|c|}{ Sucrose intake and biomarker in separate models } & \multicolumn{6}{|c|}{ Sucrose intake and biomarker in the same model } \\
\hline & \multicolumn{3}{|c|}{ BMI $\left(\mathrm{kg} / \mathrm{m}^{2}\right)$ adjusted for age } & \multicolumn{3}{|c|}{ WC $(\mathrm{cm})$ adjusted for age and height } & \multicolumn{3}{|c|}{ BMI $\left(\mathrm{kg} / \mathrm{m}^{2}\right)$ adjusted for age } & \multicolumn{3}{|c|}{ WC $(\mathrm{cm})$ adjusted for age and height } \\
\hline & $\beta$ & $95 \% \mathrm{Cl}$ & $P$ & $\beta$ & $95 \% \mathrm{Cl}$ & $P$ & $\beta$ & $95 \% \mathrm{Cl}$ & $P$ & $\beta$ & $95 \% \mathrm{Cl}$ & $P$ \\
\hline \multicolumn{13}{|l|}{ Men $(n 797)$} \\
\hline Biomarker* & 0.09 & $-0.13,0.31$ & 0.442 & 0.42 & $-0.22,1.06$ & 0.195 & 0.22 & $0.00,0.45$ & 0.052 & 0.82 & $0.17,1.46$ & 0.013 \\
\hline & \multicolumn{6}{|c|}{ Women (n 937) } & -1.30 & $-1.81,-0.79$ & $<0.001$ & -3.79 & $-5 \cdot 25,-2 \cdot 33$ & $<0.001$ \\
\hline Biomarker* & 0.40 & $0.14,0.65$ & 0.002 & 0.85 & $0.24,1.46$ & 0.007 & 0.50 & $0.25,0.76$ & $<0.001$ & $1 \cdot 12$ & $0.52,1.73$ & $<0.001$ \\
\hline Intake† & -1.60 & $-2 \cdot 25,-0.96$ & $<0.001$ & $-4 \cdot 19$ & $-5.75,-2.64$ & $<0.001$ & -1.80 & $-2 \cdot 45,-1 \cdot 15$ & $<0.001$ & -4.63 & $-6 \cdot 19,-3.07$ & $<0.001$ \\
\hline \multicolumn{13}{|c|}{ Sex-adjusted ( $n$ 1734) } \\
\hline Biomarker* & 0.25 & $0.08,0.43$ & 0.004 & & - & - & 0.38 & $0.21,0.55$ & $<0.001$ & & - & - \\
\hline Intake† & -1.40 & $-1.81,-0.99$ & $<0.001$ & & - & - & -1.57 & $-1.99,1.16$ & $<0.001$ & & - & - \\
\hline
\end{tabular}

Data are shown with biomarker and intake in separate models as well as in the same model. Regression coefficients $\beta$ and $95 \%$ confidence intervals were determined by linear regression. Biomarker and intake data were log-transformed before analysis.

*Urinary sucrose, adjusted by specific gravity.

Table 2 Associations between sucrose intake and risk of being overweight or obese after three years of follow-up at the second health check among men and women ( $n$ 1734) aged 39-77 years, Norfolk cohort of the European Prospective Investigation into Cancer and Nutrition (EPIC-Norfolk)

\begin{tabular}{|c|c|c|c|c|c|c|c|c|c|c|c|c|c|c|c|}
\hline & \multicolumn{5}{|c|}{ Men $(n 797)$} & \multicolumn{5}{|c|}{ Women ( $n$ 937) } & \multicolumn{5}{|c|}{ All $(n$ 1734) } \\
\hline & \multicolumn{2}{|c|}{ Overweight* $^{\star}$} & \multirow[b]{2}{*}{ OR } & \multirow[b]{2}{*}{$95 \% \mathrm{Cl}$} & \multirow[b]{2}{*}{$P$} & \multicolumn{2}{|c|}{ Overweight $^{\star}$} & \multirow[b]{2}{*}{ OR } & \multirow[b]{2}{*}{$95 \% \mathrm{Cl}$} & \multirow[b]{2}{*}{$P$} & \multicolumn{2}{|c|}{ Overweight $^{*}$} & \multirow[b]{2}{*}{ OR } & \multirow[b]{2}{*}{$95 \% \mathrm{Cl}$} & \multirow[b]{2}{*}{$P$} \\
\hline & $n$ & $\%$ & & & & $n$ & $\%$ & & & & $n$ & $\%$ & & & \\
\hline \multicolumn{16}{|c|}{ Urinary sucrose, adjusted by specific gravity } \\
\hline Q1 & 105 & 66 & 1.00 & Ref. & - & 106 & 56 & 1.00 & Ref. & - & 201 & 58 & 1.00 & Ref. & - \\
\hline Q2 & 114 & 72 & $1 \cdot 31$ & $0.82,2 \cdot 11$ & 0.263 & 98 & 52 & 0.84 & $0.56,1.27$ & 0.416 & 216 & 62 & 1.15 & $0.84,1.56$ & 0.384 \\
\hline Q3 & 115 & 72 & $1 \cdot 33$ & $0.83,2.14$ & 0.238 & 105 & 56 & 0.96 & $0.64,1.45$ & 0.857 & 225 & 65 & 1.26 & $0.92,1.71$ & 0.149 \\
\hline Q4 & 119 & 75 & 1.54 & $0.95,2.50$ & 0.082 & 123 & 66 & 1.45 & $0.96,2 \cdot 21$ & 0.080 & 237 & 68 & 1.43 & $1.05,1.96$ & 0.025 \\
\hline Q5 & 120 & 75 & 1.57 & $0.96,2.57$ & 0.070 & 119 & 63 & 1.33 & $0.88,2.02$ & 0.180 & 245 & 71 & 1.54 & $1 \cdot 12,2 \cdot 12$ & 0.008 \\
\hline Trend & & & $1 \cdot 11$ & $1.00,1.25$ & 0.054 & & & $1 \cdot 12$ & $1.02,1.23$ & 0.020 & & & 1.12 & $1.04,1.20$ & 0.003 \\
\hline \multicolumn{16}{|c|}{ Sucrose intake, 7DD energy-adjusted } \\
\hline Q1 & 128 & 80 & 1.00 & Ref. & - & 120 & 64 & 1.00 & Ref. & - & 255 & 73 & 1.00 & Ref. & - \\
\hline Q2 & 123 & 77 & 0.84 & $0.49,1.44$ & 0.525 & 117 & 63 & 0.92 & $0.61,1.41$ & 0.710 & 236 & 68 & 0.77 & $0.56,1.08$ & 0.129 \\
\hline Q3 & 106 & 66 & 0.48 & $0.29,0.80$ & 0.005 & 107 & 57 & 0.73 & $0.48,1.11$ & 0.146 & 209 & 60 & 0.56 & $0.41,0.78$ & 0.001 \\
\hline Q4 & 107 & 67 & 0.50 & $0.30,0.84$ & 0.008 & 103 & 55 & 0.68 & $0.45,1.03$ & 0.068 & 212 & 61 & 0.58 & $0.42,0.80$ & 0.001 \\
\hline Q5 & \multirow{2}{*}{\multicolumn{2}{|c|}{109}} & 0.53 & $0.32,0.89$ & 0.015 & \multirow{2}{*}{\multicolumn{2}{|c|}{104}} & 0.69 & $0.45,1.04$ & 0.076 & 212 & 61 & 0.56 & $0.40,0.77$ & $<0.001$ \\
\hline Trend & & & 0.84 & $0.75,0.94$ & 0.003 & & & 0.90 & $0.82,0.99$ & 0.026 & \multicolumn{2}{|l|}{ 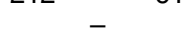 } & 0.87 & $0.81,0.93$ & $<0.001$ \\
\hline
\end{tabular}

7DD, 7 d diet diary; Q1, quintile 1 (lowest); Q2, quintile 2; Q3, quintile 3; Q4, quintile 4; Q5, quintile 5 (highest); Ref., referent category.

Odds ratios and $95 \%$ confidence intervals were determined by logistic regression, adjusted for age and sex.

*Second health check, $\mathrm{BMl}>25 \cdot 0 \mathrm{~kg} / \mathrm{m}^{2}$. 
After imputation of data below and above the calibration range with $4.9 \mu \mathrm{M}$ and $500 \cdot 1 \mu \mathrm{M}$ respectively, the urinary sucrose biomarker was significantly positively associated with self-reported sucrose intake $(\beta=0.051$; $95 \%$ CI 0.039, 0.063). The associations with BMI at followup ( $\beta=0 \cdot 17 ; 95 \%$ CI 0.06, 0.27; adjusted for age and sex) and WC at follow-up up (men: $\beta=0.55 ; 95 \%$ CI 0.15, 0.95; women: $\beta=0.54 ; 95 \% \mathrm{CI} 0 \cdot 15,0 \cdot 93$; both adjusted for age) were also significant. The association between the biomarker and risk of being overweight was also significant, with an OR of 1.08 (95\% CI 1.01, 1.14; adjusted for age and sex) per log increase of biomarker; the OR when using dietary data was 0.61 (95\% CI $0.51,0.72$; adjusted for age and sex).

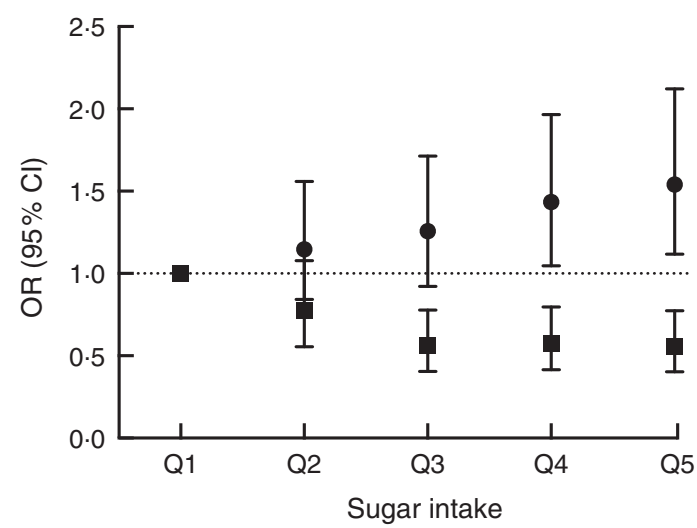

Fig. 3 Association between sucrose intake and risk of overweight or obesity after three years of follow-up using either dietary data ( $\mathbf{\square}$, energy-adjusted, as assessed by $7 \mathrm{~d}$ diet diary) or biomarker (0, urinary sucrose, adjusted by specific gravity) among men and women ( $n$ 1734) aged 39-77 years, Norfolk cohort of the European Prospective Investigation into Cancer and Nutrition (EPIC-Norfolk). Presented are odds ratios with their $95 \%$ confidence intervals represented by vertical bars (Q1, quintile 1 (lowest); Q2, quintile 2; Q3, quintile 3; Q4, quintile 4; Q5, quintile 5 (highest))

\section{Sensitivity analysis with gastric ulcer status}

A diagnosis of gastric ulcer was reported by sixty-seven participants (thirty-seven men, thirty women) and they did not differ significantly in age and BMI (baseline and second health check). Urinary sucrose concentration was significantly different in men (two-sided $t$ test with logtransformed data, geometric mean (SD); gastric ulcer $v$. other: 53 ( $\mathrm{sD} 1) \mu \mathrm{m} v .36$ ( $\mathrm{sD} 1) \mu \mathrm{M} ; P=0.04$ ) but not in women (26 (SD 1) $\mu \mathrm{M} v .27$ (SD 1) $\mu \mathrm{M} ; P=0 \cdot 8$ ). Participants with self-reported gastric ulcer were not equally distributed across quintiles of biomarker $\left(P=0.004, \chi^{2}\right.$ test), with most participants found in the fourth $(n 22)$, fifth ( $n$ 18) and first ( $n$ 14) quintiles.

The associations between biomarker and self-reported energy-adjusted sucrose intake, BMI and WC at the second health check were not materially different after excluding participants with self-reported gastric ulcer (see online supplementary material, Supplemental Table 9). The estimated risk of being overweight or obese after follow-up was slightly attenuated, yet the results were not materially different (see online supplementary material, Supplemental Table 10).

\section{Discussion}

In the present study, we investigated prospectively the risk of overweight and obesity in relation to sucrose intake estimated by 7DD and biomarker. Using urinary sucrose as the measure of sucrose intake, participants in the highest $v$. the lowest quintile for sucrose intake had $54 \%$ greater risk of being overweight or obese. In contrast, using self-reported sucrose intake measured by 7DD, those in the highest $v$. the lowest quintile for self-reported sucrose intake were at $44 \%$ lower risk of being overweight or obese.

Table 3 Comparison of different assessment methods: associations (regression coefficients $\beta$ and $95 \%$ confidence intervals) between sucrose intake (log-transformed) and BMI and waist circumference (WC) after three years of follow-up among men and women ( $n$ 1734) aged 39-77 years, Norfolk cohort of the European Prospective Investigation into Cancer and Nutrition (EPIC-Norfolk)

\begin{tabular}{|c|c|c|c|c|c|c|}
\hline & \multirow{2}{*}{\multicolumn{2}{|c|}{$\frac{\text { BMI }\left(\mathrm{kg} / \mathrm{m}^{2}\right)^{\star}, \dagger}{\text { All }}$}} & \multicolumn{4}{|c|}{ WC $(\mathrm{cm})^{*}, \ddagger$} \\
\hline & & & \multicolumn{2}{|c|}{ Men } & \multicolumn{2}{|c|}{ Women } \\
\hline & $\beta$ & $95 \% \mathrm{Cl}$ & $\beta$ & $95 \% \mathrm{Cl}$ & $\beta$ & $95 \% \mathrm{Cl}$ \\
\hline $\begin{array}{l}\text { 7DD (adjusted for energy intake) } \\
\text { 24HDR§ (adjusted for energy intake) } \\
\text { FFQ (adjusted for energy intake) } \\
\text { 7DD } \\
\text { 24HDR } \\
\text { FFQ } \\
\text { Biomarkerll }\end{array}$ & $\begin{array}{r}-1.40 \\
-0.78 \\
-1.32 \\
-1.38 \\
-0.80 \\
-1.07 \\
0.25\end{array}$ & $\begin{array}{l}-1.81,-0.99 \\
-1.06,-0.51 \\
-1.83,-0.81 \\
-1.72,-1.04 \\
-1.04,-0.57 \\
-1.42,-0.71 \\
0.08,0.43\end{array}$ & $\begin{array}{r}-3 \cdot 34 \\
-2 \cdot 31 \\
-2 \cdot 74 \\
-2 \cdot 80 \\
-2 \cdot 03 \\
-2 \cdot 76 \\
0.43\end{array}$ & $\begin{array}{l}-4 \cdot 77,-1.91 \\
-3.33,-1.30 \\
-4.54,-0.94 \\
-4.00,-1.61 \\
-2.92,-1.13 \\
-4.03,-1.49 \\
-0.21,1.06\end{array}$ & $\begin{array}{r}-4 \cdot 21 \\
-2 \cdot 14 \\
-2.52 \\
-3.85 \\
-2.05 \\
-1.87 \\
0.87\end{array}$ & $\begin{array}{l}-5 \cdot 77,-2 \cdot 66 \\
-3 \cdot 13,-1 \cdot 15 \\
-4 \cdot 44,-0.59 \\
-5 \cdot 14,-2 \cdot 56 \\
-.288,-1 \cdot 22 \\
-3 \cdot 19,-0.54 \\
0.26,1.49\end{array}$ \\
\hline
\end{tabular}

7DD, $7 \mathrm{~d}$ diet diary; $24 \mathrm{HDR}, 24 \mathrm{~h}$ recall.

${ }^{*}$ At second health check.

†Adjusted for age and sex.

$\ddagger$ Adjusted for height and age.

§Among 1685 participants only.

॥Urinary sucrose adjusted by specific gravity. 
Our results show a clear discrepancy in the association between sucrose intake and the risk of overweight or obesity depending on the dietary assessment method. The associations observed with self-reported intake are in agreement with data from cross-sectional studies published previously ${ }^{(3,28)}$, in which a cross-sectional analysis of 875 participants of EPIC-Norfolk found a strong positive association between sugar intake and obesity risk when using the biomarker (trend per quintile, normal weight $v$. obese: $\mathrm{OR}=1 \cdot 26 ; 95 \%$ CI 1.14, $1.40 ; P<0.0001)^{(3)}$.

There are several possible reasons for this apparent discrepancy. The inverse association between selfreported sucrose intake and body weight has been used as a basis for dietary recommendations ${ }^{(7)}$. First the suitability of the biomarker used in the present study needs consideration: while there is only limited information on the physiological mechanisms underlying the absorption and excretion of sucrose, several controlled-feeding studies have shown a clear dose-response relationship between sucrose intake and urinary excretion ${ }^{(12-14)}$, which is independent of $\mathrm{BMI}^{(15)}$. This has led to the development and validation of $24 \mathrm{~h}$ urinary sucrose and fructose as a biomarker for total sugar intake ${ }^{(16)}$. However, $24 \mathrm{~h}$ urine samples are difficult to obtain and are cost-prohibitive in large observational studies, such as EPIC-Norfolk. Sucrose concentration in spot urine samples can be used as a concentration marker ${ }^{(29)}$ in order to rank participants according to intake. Given that we used spot urine samples to measure the biomarker, these samples required an adjustment for urine dilution. We used specific gravity, rather than urinary creatinine concentration, which is commonly used for this purpose. In our participants, urinary creatinine was highly significantly associated with BMI $(P<0.0001)$ and therefore would have had a strong confounding effect on the observed association. In the previous analysis of EPIC-Norfolk, the ratio of urinary sucrose to fructose was used to adjust for the dilution effect in spot urine samples ${ }^{(3)}$. However, while this ratio was strongly associated with sugar intake and obesity risk, it is difficult to interpret as urinary fructose concentration depends not only on fructose but also on sucrose consumption, as sucrose is hydrolysed into glucose and fructose in vivo. In the present study we have therefore focused on urinary sucrose only and used urinary specific gravity to compensate for the dilution effect, although risk estimates remained similar when using sucrose:fructose as a measure of intake. While specific gravity is also associated with BMI, the association was weaker than for creatinine $(\rho=0.08 ; P=0.0004)$. We could show that specific-gravity-adjusted urinary sucrose concentration was positively, and significantly, associated with selfreported intake from 7DD, $24 \mathrm{HDR}$ and FFQ.

Our results indicate that those in the highest category of sucrose intake as measured by the biomarker had the highest risk of being overweight or obese after three years of follow-up. Furthermore, we observed that a combination of
Table 4 Main sources of sucrose intake in men $(n 797)$ and women ( $n$ 937) aged 39-77 years, Norfolk cohort of the European Prospective Investigation into Cancer and Nutrition (EPIC-Norfolk), based on data from $7 \mathrm{~d}$ diaries

\begin{tabular}{|c|c|c|c|}
\hline & Mean & SD & Contribution (\%) \\
\hline \multicolumn{4}{|l|}{ Men } \\
\hline Table sugar & $16 \cdot 8$ & $25 \cdot 3$ & 29 \\
\hline Cake & 8.9 & $9 \cdot 8$ & 15 \\
\hline Fruits & $6 \cdot 2$ & 5.9 & 11 \\
\hline Sweet biscuits & $3 \cdot 7$ & 4.9 & 6 \\
\hline Pudding (milk-based) & $2 \cdot 8$ & $3 \cdot 6$ & 5 \\
\hline Confectionery (chocolate) & $2 \cdot 8$ & 5.4 & 5 \\
\hline Breakfast cereals & $2 \cdot 0$ & 3.6 & 3 \\
\hline Jam and marmalade & 1.7 & $3 \cdot 8$ & 3 \\
\hline Squash and lemonade & 1.5 & 4.4 & 3 \\
\hline Pudding (cereal-based) & 1.5 & 3.7 & 3 \\
\hline \multicolumn{4}{|l|}{ Women } \\
\hline Fruits & 7.5 & $5 \cdot 8$ & 18 \\
\hline Table sugar & 6.5 & $14 \cdot 1$ & 15 \\
\hline Cake & $6 \cdot 3$ & $6 \cdot 8$ & 15 \\
\hline Confectionery (chocolate) & $2 \cdot 9$ & $5 \cdot 0$ & 7 \\
\hline Sweet biscuits & $2 \cdot 8$ & 3.4 & 7 \\
\hline Pudding (milk-based) & $2 \cdot 3$ & 3.0 & 5 \\
\hline Yoghurt & 1.8 & $3 \cdot 2$ & 4 \\
\hline Breakfast cereals & 1.6 & $2 \cdot 6$ & 4 \\
\hline Confectionery (non-chocolate) & 1.4 & 3.5 & 3 \\
\hline Juices & 1.4 & $2 \cdot 3$ & 3 \\
\hline
\end{tabular}

Mean and standard deviation of intake in $\mathrm{g} / \mathrm{d}$ and percentage contribution to total sucrose intake.

low self-reported sucrose intake and high biomarker was associated with high BMI and WC. The tendency to underreport the intake of unhealthy foods and foods with high sugar content, especially among those who are overweight and obese ${ }^{(9)}$, may possibly be reason for the observed inverse association with self-reported intake. The positive association between BMI and the ratio of biomarker to selfreported intake suggests that participants with a higher BMI are more likely to under-report intake. As we have shown previously, the relationship between dietary and urinary sucrose is not affected by $\mathrm{BMI}^{(15)}$ and therefore does not explain this observation. There is some evidence suggesting that an important cause of under-reporting is the failure to report snack foods consumed between meals ${ }^{(11)}$ and biscuits, cakes, confectionery and other types of snacks were main contributors to total sucrose intake in EPICNorfolk (Table 4). A recent biomarker-based study detected substantial measurement error in self-reported sugar intake assessed by both FFQ and 24HDR, which was greater in women than men for both dietary assessment instruments ${ }^{(16)}$.

The strengths of the present study are the use of a biomarker as a surrogate measure of sucrose intake besides self-reported intake only, use of 7DD as a more detailed self-report dietary instrument and the wellcharacterised cohort. We also report findings for FFQ and $24 \mathrm{HDR}$. However, there were also some limitations: sensitivity of our method for quantifying sucrose and fructose in urine was limited, which resulted in a reduced sample size and the pseudo-random selection of samples. 
Yet, analyses with imputed values for those outside the calibration range suggest that the reduced concentration range did not affect the observed associations. We also used spot urines, rather than $24 \mathrm{~h}$ urine collection, to measure sucrose and fructose. Furthermore, we report $P$ values without adjustment for multiple testing and this must be taken into consideration when interpreting results. There were also some participants with gastric damage, which can lead to increased permeability of gastric mucosa for sucrose and increased excretion in urine $^{(30)}$. However, gastric damage did not affect the outcome of our analyses.

There is some ambiguity in data from observational studies investigating associations between sugar intake and BMI. While sugar-sweetened beverage intake is clearly associated with an increased $\mathrm{BMI}^{(4,5,31)}$, the association is less clear for total sugar intake and sucrose and some studies report inverse associations. Indeed, recent guidance by the European Food Safety Authority ${ }^{(7)}$ suggests such an association. In the present study we observed a clear discrepancy between self-reported sucrose intake and biomarker-based findings in the relationship with BMI, despite correlation between the two measures of sugar intake. This is consistent with our previous results ${ }^{(3)}$. These data suggest that the inconsistency of data on sucrose and obesity may be in part attributed to misreporting and nutritional biomarkers are important to understand these associations better.

\section{Acknowledgements}

Acknowledgements: The authors thank all EPIC-Norfolk study participants and staff for their contribution to the study. They also thank the NIHR BRC-MRC BioRepository at the Cambridge Biomedical Campus for providing infrastructure and equipment for sample preparation. Financial support: This project was supported by the Word Cancer Research Fund (WCRF), Cancer Research UK and the Medical Research Council (MRC). WCRF, Cancer Research UK and MRC had no role in the design, analysis or writing of this article. Conflict of interest: None. Authorship: The responsibilities of the authors were as follows: G.G.C.K. developed the analytical method, conducted the statistical analyses, wrote the manuscript and had primary responsibility for the final content; N.T. conducted statistical analyses and contributed to the manuscript; J.L.G., M.A.S., L.R. and S.M.A. developed the analytical method and conducted sample analyses; M.A.H.L. and A.A.M. were responsible for dietary data analysis and contributed to the manuscript; R.N.L. was responsible for follow-up and data processing; K.-T.K. (principal investigator of EPIC-Norfolk) contributed to the manuscript. All authors read and approved the final manuscript. Ethics of buman subject participation: The study received ethical approval by the Norwich District
Health Authority Ethics Committee and all participants gave signed informed consent.

\section{Supplementary material}

To view supplementary material for this article, please visit http://dx.doi.org/10.1017/S1368980015000300

\section{References}

1. World Cancer Research Fund/American Institute of Cancer Research (2007) Food, Nutrition, Physical Activity, and the Prevention of Cancer: A Global Perspective. Washington, DC: AICR.

2. Goh LG, Dhaliwal SS, Welborn TA et al. (2014) Anthropometric measurements of general and central obesity and the prediction of cardiovascular disease risk in women: a crosssectional study. BMJ Open 4, e004138.

3. Bingham SA, Luben RN, Welch AA et al. (2007) Epidemiologic assessment of sugars consumption using biomarkers: comparisons of obese and nonobese individuals in the European prospective investigation of cancer Norfolk. Cancer Epidemiol Biomarkers Prev 16, 1651-1654.

4. Malik VS, Pan A, Willett WC et al. (2013) Sugar-sweetened beverages and weight gain in children and adults: a systematic review and meta-analysis. Am J Clin Nutr 98, 1084-1102.

5. Te Morenga L, Mallard S, Mann J (2013) Dietary sugars and body weight: systematic review and meta-analyses of randomised controlled trials and cohort studies. BMJ 346 , e7492.

6. Institute of Medicine, Panel on Macronutrients, Standing Committee on the Scientific Evaluation of Dietary Reference Intakes (2005) Dietary Reference Intakes for Energy, Carbohydrate, Fiber, Fat, Fatty Acids, Cholesterol, Protein, and Amino Acids. Washington, DC: National Academies Press.

7. European Food Safety Authority, Panel on Dietetic Products, Nutrition, and Allergies (2010) Scientific opinion on dietary reference values for carbohydrates and dietary fibre. EFSAJ 8, 1462-1539.

8. Johansson L, Solvoll K, Bjorneboe GE et al. (1998) Underand overreporting of energy intake related to weight status and lifestyle in a nationwide sample. Am J Clin Nutr $\mathbf{6 8}$, 266-274.

9. Pryer JA, Vrijheid M, Nichols R et al. (1997) Who are the 'low energy reporters' in the dietary and nutritional survey of British adults? Int J Epidemiol 26, 146-154.

10. Bingham SA, Cassidy A, Cole T et al. (1995) Validation of weighed records and other methods of dietary assessment using the $24 \mathrm{~h}$ urine nitrogen technique and other biological markers. Br J Nutr 73, 531-550.

11. Poppitt SD, Swann D, Black AE et al. (1998) Assessment of selective under-reporting of food intake by both obese and non-obese women in a metabolic facility. Int J Obes Relat Metab Disord 22, 303-311.

12. Nakamura H \& Tamura Z (1972) Gas chromatographic analysis of mono- and disaccharides in human blood and urine after oral administration of disaccharides. Clin Chim Acta 39, 367-381.

13. Luceri C, Caderni G, Lodovici M et al. (1996) Urinary excretion of sucrose and fructose as a predictor of sucrose intake in dietary intervention studies. Cancer Epidemiol Biomarkers Prev 5, 167-171.

14. Tasevska N, Runswick SA, McTaggart A et al. (2005) Urinary sucrose and fructose as biomarkers for sugar consumption. Cancer Epidemiol Biomarkers Prev 14, 1287-1294. 
15. Joosen AMCP, Kuhnle GGC, Runswick SA et al. (2008) Urinary sucrose and fructose as biomarkers of sugar consumption: comparison of normal weight and obese volunteers. Int J Obes (Lond) 32, 1736-1740.

16. Tasevska N, Midthune D, Potischman N et al. (2011) Use of the predictive sugars biomarker to evaluate self-reported total sugars intake in the Observing Protein and Energy Nutrition (OPEN) study. Cancer Epidemiol Biomarkers Prev 20, 490-500.

17. Day NE, Oakes S, Luben RN et al. (1999) EPIC-Norfolk: study design and characteristics of the cohort. European Prospective Investigation of Cancer. Br J Cancer 80, Suppl. 1, S95-S103.

18. Hayat SA, Luben R, Keevil VL et al. (2013) Cohort profile: a prospective cohort study of objective physical and cognitive capability and visual health in an ageing population of men and women in Norfolk (EPIC-Norfolk 3). Int J Epidemiol 43, 1063-1072.

19. Welch AA, McTaggart A, Mulligan AA et al. (2001) DINER (Data Into Nutrients for Epidemiological Research) - a new data-entry program for nutritional analysis in the EPICNorfolk cohort and the 7-day diary method. Public Health Nutr 4, 1253-1265.

20. Lentjes MAH, McTaggart A, Mulligan AA et al. (2014) Dietary intake using 7-day diaries in British men and women in the EPIC-Norfolk Study: a focus on methodological issues. $\mathrm{Br} \mathrm{J}$ Nutr 111, 516-526.

21. Mulligan AA, Luben RN, Bhaniani A et al. (2014) A new tool for converting food frequency questionnaire data into nutrient and food group values: FETA research methods and availability. BMJ Open 4, e004503.

22. Wareham NJ, Jakes R, Rennie K et al. (2002) Validity and repeatability of the EPIC-Norfolk physical activity questionnaire. Int J Epidemiol 31, 168-174.
23. Park JY, Mitrou PN, Keogh RH et al. (2012) Self-reported and measured anthropometric data and risk of colorectal cancer in the EPIC-Norfolk study. Int J Obes (Lond) 36, $107-118$

24. Kuhnle GG, Joosen AM, Wood TR et al. (2008) Detection and quantification of sucrose as dietary biomarker using gas chromatography and liquid chromatography with mass spectrometry. Rapid Commun Mass Spectrom 22, 279-282.

25. Atherton H, Bailey N, Zhang W et al. (2006) A combined $1 \mathrm{H}-\mathrm{NMR}$ spectroscopy- and mass spectrometry-based metabolomic study of the PPAR- $\alpha$ null mutant mouse defines profound systemic changes in metabolism linked to the metabolic syndrome. Physiol Genomics 27, 178-186.

26. von Elm E, Altman DG, Egger $M$ et al. (2007) The Strengthening the Reporting of Observational Studies in Epidemiology (STROBE) statement: guidelines for reporting observational studies. Lancet 370, 1453-1457.

27. Core Team R (2014) R: A Language and Environment for Statistical Computing. Vienna: R Foundation for Statistical Computing.

28. Bolton-Smith C \& Woodward M (1994) Dietary composition and fat to sugar ratios in relation to obesity. Int J Obes Relat Metab Disord 18, 820-828.

29. Jenab M, Slimani N, Bictash M et al. (2009) Biomarkers in nutritional epidemiology: applications, needs and new horizons. Hum Genet 125, 507-525.

30. Sutherland LR, Verhoef M, Wallace JL et al. (1994) A simple, non-invasive marker of gastric damage: sucrose permeability. Lancet 343, 998-1000.

31. Emond JA, Patterson RE, Jardack PM et al. (2014) Using doubly labeled water to validate associations between sugar-sweetened beverage intake and body mass among White and African-American adults. Int J Obes (Lond) 38, 603-609. 